Macedonian Pharmaceutical Bulletin, 66 (Suppl 1) 121 - 122 (2020)

Online ISSN 1857 - 8969

UDC: 615.015:[547.962.1:620.3

DOI: 10.33320/maced.pharm.bull.2020.66.03.060

Short communication

\title{
An introduction of a new generation of Proticles
}

\author{
Katja Fresacher ${ }^{1}$, Bettina Huemer ${ }^{1}$, Martin Reiser ${ }^{1}$, Andreas Zimmer ${ }^{1}$ \\ Institute of Pharmaceutical Sciences, Department of Pharmaceutical Technology and Biopharmacy, \\ Karl-Franzens-University Graz, Universitätsplatz 1, 8010 Graz, Austria
}

\section{Introduction}

MicroRNAs (miRs) used as active ingredients were put more often into spotlight these days. In their application field, of course, there have to be some obstacles to overcome: On one hand there is the low bioavailability and cellular uptake and on the other hand affinity for enzymatic degradation (Blanco et al., 2015). Therefore, protamineoligonucleotide-nanoparticles, so-called Proticles, basically consisting of miRs and protamine, a cationic peptide, represent a new generation of nanoparticulate (NP) Drug Delivery Systems (DDS) to deal with that challenges (Scheicher et al., 2015). Proticles are formed by a self-assembling mechanism due to electrostatic interactions between the positively charged protamine and the negatively charged miR (Junghans et al., 2000). To mediate the $\mathrm{miR}$ release profile and decrease the strength of the electrostatic interactions the strategy of functionalizing NPs with citric acid (CA) was investigated.

\section{Materials and methods}

Materials

miR miRIDIAN ${ }^{\mathrm{TM}}$ microRNA mimic negative control was purchased from Dharmacon (Lafayette, Colorado), protamine (free base) from Sigma Aldrich (St. Louis, Missouri) and citric acid from Caesar \& Loretz GmbH (Hilden, Germany).

\section{Nanoparticle preparation}

All NPs were prepared in aqueous solutions by mixing miR and protamine, final miR concentration was $50 \mu \mathrm{g} / \mathrm{mL}$. The mass ratio of miR and protamine (1:3) was determined experimentally (data not shown). Aqueous CA solution was added to the protamine solution in molar ratios from 1:1 to $1: 20$ before it was put to the miR solution.

\section{Nanoparticle characterization}

By using photon correlation spectroscopy (PCS) (Zetasizer Nano Series, Malvern Instruments, Herrenberg, Germany) the physicochemical properties of the different NP formulations were determined. Results are expressed as hydrodynamic particle diameter (Z-average), polydispersity index (PDI), in correlation to particle size distribution (PSD), and the surface charge as Zetapotential, which is an indicator for NP stability.

For further stability studies we developed a stability assay to determine the decrease in stability by adding $50 \mu \mathrm{L} 0.01 \mathrm{M} \mathrm{NaOH}$ to the NP solutions and detect the total number of remaining NPs afterwards with PCS. The Proticle dispersions were prepared as described before and afterwards treated with $0.01 \mathrm{M} \mathrm{NaOH}$ and incubated for $1 \mathrm{~h}$ in a thermomixer $\left(60{ }^{\circ} \mathrm{C} ; 1000 \mathrm{rpm}\right)$ before the assay.

\section{Drug loading}

The drug loading efficiencies were determined by an indirect quantification method using reversed

\footnotetext{
* katja.fresacher@uni-graz.at
} 
phase high performance liquid chromatography (RPHPLC; Agilent 1260 Infinity, Agilent technologies, Santa Clara, California). An Agilent PLRP-S $50 \times 2.1 \mathrm{~mm}$ column $(3 \mu \mathrm{m}, 300 \AA)$ was used at $80{ }^{\circ} \mathrm{C}$. Mobile phase A was $0.1 \mathrm{M}$ Triethylammonium acetate buffer (TEAA), whereas mobile phase $\mathrm{B}$ was acetonitrile. A gradient was used with a flowrate of $0.2 \mathrm{~mL} / \mathrm{min}$. It was starting with $5 \% \mathrm{~B}$ for $1 \mathrm{~min}$ and increased to $35 \% \mathrm{~B}$ in 7 min afterwards. For detection a diode array UV absorption detector at $254 \mathrm{~nm}$ was used. The NP dispersions were centrifuged for two hours at $4{ }^{\circ} \mathrm{C}$ and $20000 \mathrm{rcf}, 10 \mu \mathrm{L}$ of the supernatant was injected for quantification. Bound $\mathrm{miR}$ was calculated as the difference between the amount of used $\mathrm{miR}$ and the detected $\mathrm{miR}$ in percentage.

\section{Results and discussion}

\section{Nanoparticle characterization}

The addition of CA to the Proticles had no influence on their size $(\sim 110 \mathrm{~nm})$ except sample 1:5. It is believed that this molar ratio totally neutralizes the positive functions of protamine which lead to a kind of steady state. However, the PDIs $(<0.2)$ represent monodisperse NP populations as well as stable DDSs. The Zetapotential of the NPs without any CA was $31.32 \pm 1.32 \mathrm{mV}$. By increasing the CA content, a decreasing trend was observed, which correlates with a decrease in stability. Interestingly, the result of the twentyfold surplus is increasing again which can be explained by rising protonation of the system. The detected derived count rate ( $\mathrm{dCR})$ of NP dispersions is expressed as percentage of remaining NPs after the treatment with $0.01 \mathrm{M}$ $\mathrm{NaOH}$. As a $100 \%$ reference the dCR of Proticles without any $\mathrm{CA}$ and an addition of $\mathrm{H}_{2} \mathrm{O}$ instead of $\mathrm{NaOH}$ was taken. The results highlight a significant decrease $(p<0.05)$ in number of NPs which correlates with a decrease in stability. These formulations, which contain a ten- or twentyfold surplus of CA represent the lowest results and therefore the biggest lost in stability ( $94 \%$ loss). One possible explanation for this observation could be protonation. Based on high proton concentrations due to acid addition the NPs started to repulse each other and therefore the interaction between the NPs is lower than for NPs with less acid functions.

\section{Drug loading}

With a RP-HPLC system the drug load capacity of the Proticles was visualized and determined. Therefore, all tested formulations presented a drug load of $>92 \%$, no differences due to CA ratios could be found. These results give us important information about the preparation procedure - which seems to be well working - and the possible capacity of the advanced Proticles concerning drug load and transport efficacy. High binding affinities may help to improve a successful and sufficient dissociation rate of $\mathrm{miR}$ in further experiments.

\section{Conclusion}

The aim of this work was to characterize advanced Proticles, which basically consist of the positively charged protamine and a negatively charged miR supplemented with CA. The formation of NPs occurs immediately by mixing the protamine solution and the miR solution due to electrostatic interaction between the two components. We observed no difference in particle size, except NPs with a fivefold surplus of CA. A monodisperse PSD over all formulations was found. Due to increasing $\mathrm{CA}$ ratios the Zetapotential was decreasing, which lead to the assumption that higher CA contents destabilize the Proticles. These findings could be supported with the results of the self-developed $\mathrm{NaOH}$ assay. By applying $\mathrm{NaOH}$ to the NPs a statistically significant $(\mathrm{p}<0.05)$ stability reduction (up to $\sim 94 \%$ ) was observed due to addition of CA.

A miR binding capacity of $>92 \%$ independent from CA ratios has been found, which demonstrated a well working and robust preparing procedure. All in all, this new approach of DDSs constitutes a very promising candidate to deal with the difficulties of miR drug delivery and cellular drug release.

\section{References}

Blanco, E., Shen, H., Ferrari, M., 2015. Principles of nanoparticle design for overcoming biological barriers to drug delivery. Nat. Biotechnol. 33, 941951.

Junghans, M., Kreuter, J., Zimmer, A., 2000. Antisense delivery using protamine-oligonucleotide particles. Nucleic Acids Res. 28, E45.

Scheicher, B., Schachner-Nedherer A.L., Zimmer, A., 2015. Protamine-oligonucleotide-nanoparticles: Recent advances in drug delivery and drug targeting. Eur. J. Pharm. Sci. 75, 54-59.

Maced. Pharm. Bull. 66 (Suppl 1) 121 - 122 (2020) 